ADRIANA BRUNO ${ }^{1}$

Karina SerRavalle ${ }^{2}$

Ana Gabriela Travassos 3

Bruno GIL de Carvalho Lima ${ }^{4}$

\title{
Distribuição dos genótipos de papilomavírus humano em mulheres do estado da Bahia, Brasil
}

\author{
Genotype distribution of buman papillomavirus \\ in women from the state of Babia, Brazil
}

Artigo Original

Palavras-chave
Pappilomavirus humano
Mulheres
Genótipo
Infecções por Papillomavirus/diagnóstico
Bahia
Brasil
Keywords
Human papillomavirus
Women
Genotype
Papillomavirus infections/diagnosis
Bahia
Brazil

Brazil

\section{Resumo}

OBJETIVO: $\bigcirc$ objetivo foi descrever a distribuição dos genótipos do papilomavírus humano e a frequência de infecções por múltiplos genótipos, bem como avaliar a associação entre genótipos de papilomavírus humano, faixa etária e resultados cito-histopatológicos. MÉTODOS: Estudo retrospectivo de corte transversal realizado entre junho de 2010 e outubro de 2013 em Salvador, Bahia, Brasil. Foram revisados 351 prontuários de mulheres com genotipagem positiva pelo teste PapilloCheck ${ }^{\circledR}$ usado para detectar 24 tipos de papilomavírus humano. Os achados cito-histopatológicos foram classificados em grupos de: achados negativos para neoplasia (exames citopatológico e histopatológico negativos), lesão de baixo grau (achado citopatológico - lesão intraepitelial de baixo grau - ou achado histopatológico neoplasia intraepitelial cervical grau 1, neoplasia intraepitelial vaginal grau 1 ou condiloma e lesão de alto grau lachado citopatológico - lesão intraepitelial de alto grau - ou histopatologia com laudo maior ou igual a neoplasia intraepitelial cervical grau 2 ou neoplasia intraepitelial vaginal grau 2). RESULTADOS: $\bigcirc$ genótipo de alto risco mais frequente foi o HPV 16, com 18,5\%; intervalo de confiança de 95\% (IC95\%) 14,6-23,0, seguido pelo HPV 56 (14\%; IC95\% 10,5-18,0) e o HPV 39 (13,4\%; IC95\% 9,5-16,8). O HPV 18 (5,4\%; IC95\% 3,3-8,3) esteve entre os menos comuns. Entre os tipos de baixo grau, o HPV 42 (15,7\%; IC 95\% 12,0-20,0), o HPV $6(1$ 1,4\%; IC95\% 8,3-15,2) e o HPV 44/55 (1 1, 1\%; IC95\% 8,0-14,9) foram os mais encontrados, enquanto o HPV 11 (2,8\%; IC95\% 1,4-5,2) foi o menos frequente. A proporção do HPV 16 aumentou com a severidade das anormalidades cito-histopatológicas de 13,8\% (12/87) nas lesões de baixo grau para 42,4\% (14/33) nas lesões de alto grau. Houve associação significativa entre a presença de lesão cito-histopatológica de baixo ou alto grau e os genótipos de alto risco, HPV 16, HPV 52, HPV 73 e HPV 82, e o de baixo risco, HPV 43. Mulheres com menos de 30 anos apresentaram frequência significativamente maior do HPV $16(22,2$ versus $12,9 \%, p=0,01)$, do HPV $42(19,7$ versus $10,9 \%, p=0,01)$ e do HPV $45(6,6$ versus $1,4 \%$, $\mathrm{p}=0,01$ ), além de infecção múltipla $(58,1$ versus $47,4 \%, p=0,04)$. CONCLUSÕES: Observou-se uma variabilidade da distribuição dos diversos genótipos de papilomavírus humano em mulheres no estado da Bahia. Na amostra estudada, - HPV 16 foi o mais frequente, assim como em outras regiões do Brasil e do mundo. Encontramos o HPV 56 e o HPV 39 como o segundo e o terceiro mais frequentes. Entretanto, o HPV 18 esteve entre os menos comuns. Os tipos não oncogênicos, HPV 42, 6 e 44/55, foram os mais observados, enquanto o HPV 11 foi o menos frequente.

\section{Abstract}

PURPOSE: The aim of this study was to evaluate the human papillomavirus genotypes and the frequency of multiple human papillomavirus infections, as well as to assess the association between human papillomavirus genotype, cyto-histopathological abnormalities and age range. METHODS: A retrospective cross-sectional study was carried out between June 2010 and October 2013 in Salvador, Bahia, Brazil. We analyzed 351 results of positive human papillomavirus genotyping performed using the PapilloCheck ${ }^{\circledR}$ test, designed to detect 24 human papillomavirus types. The cyto-histopathological abnormalities were classified as negative (negative cytology

\section{Correspondência \\ Adriana Bruno \\ Rua Baependi, 132 - Ondina \\ (EPP: $40170-070$ \\ Salvador (BA), Brasil \\ Recebido \\ $31 / 03 / 2014$}

Aceito com modificacōes

$31 / 07 / 2014$
Clínica IDEM - Vera Harfush; Escola Bahiana de Medicina e Saúde Pública - Salvador (BA), Brasil.

'Universidade Federal da Bahia; Clínica IDEM - Vera Harfush - Salvador (BA), Brasil.

${ }^{2}$ Setor de Biologia Molecular, Laboratório Studart \& Studart; Clínica IDEM - Vera Harfush - Salvador (BA), Brasil.

${ }^{3}$ Centro Estadual Especializado em Diagnóstico, Assistência e Pesquisa - CEDAP, Ambulatório Especializado em Saúde da Mulher;

Clínica IDEM - Vera Harfush - Salvador (BA), Brasil.

${ }^{4}$ Escola Bahiana de Medicina e Saúde Pública - Salvador (BA), Brasil.

Conflito de interesses: não há. 
and histopathology), low-grade lesions (cytologic low-grade squamous intraepithelial lesion diagnosis or histopathologic cervical intraepithelial neoplasia 1 or vaginal intraepithelial neoplasia 1 diagnosis) and high-grade lesions (cytologic high-grade squamous intraepithelial lesion diagnosis or histopathologic cervical intraepithelial neoplasia 2+ or vaginal intraepithelial neoplasia 2+ diagnosis). RESULTS: The most frequently detected high risk human papillomavirus genotype was HPBV 16, with 18.5\%, 95\% confidence interval (95\%Cl) 14.6-23.0, followed by HPV 56 (14\%; $95 \%$ Cl 10.5-18.0) and HPV $39(13.4 \% ; 95 \% \mathrm{Cl} 9.5-16.8)$. HPV 18 (5.4\%; 95\% $\mathrm{Cl} 3.3-8.3)$ was among the least frequent types. Among the low risk types, HPV 42 (15.7\%; 95\% Cl 12.0-20.0), HPV 6 (11.4\%; 95\%Cl 8.3-15.2) and HPV 44/55 (1 1.1\%; 95\%Cl 8.0-14.9) were the most frequent, while HPV 11 (2.8\%; $95 \% \mathrm{Cl} 1.4-5.2)$ was the least common. The proportion of HPV 16-positive women increased with severity of cytohistopathological abnormalities: 13.8\% (12/87) in low-grade lesion and 42.4\% (14/33) in high-grade lesion. There was association between low- or high-grade cyto-histopathological lesion and the high risk genotypes, HPV 16, HPV 52, HPV 73 and HPV 82, and the low risk type, HPV 43. Women under 30 years showed a significantly higher frequency of HPV $16(22.2$ versus $12.9 \%, p=0.01)$, HPV 42 (19.7 versus $10.9 \%, p=0.01$ ) and HPV $45(6.6$ versus $1.4 \%, p=0.01)$, and multiple human papillomavirus infections $(58.1$ versus $47.4 \%, p=0.04)$. CONCLUSIONS: We observed variability of human papillomavirus genotype distribution in women from the state of Bahia. HPV 16 was the most frequently detected high risk human papillomavirus, as also reported for other geographic areas of Brazil and for the world in general. HPV 56 and HPV 39 were the second and the third most common genotypes, whereas HPV 18 was among the least frequent types. HPV 42, 6 and 44/55 were the most frequently detected low risk human papillomavirus, and HPV 11 was the least common.

\section{Introdução}

O papilomavírus humano (HPV) é um dos agentes que com mais frequência causa doenças sexualmente transmissíveis (DST) no mundo. Estima-se que $80 \%$ das mulheres sexualmente ativas entrem em contato com o vírus em algum momento da vida ${ }^{1}$. A associação entre a persistência da infecção pelo HPV oncogênico e o carcinoma cervical está bem estabelecida ${ }^{2}$. Anualmente, 270 mil mulheres morrem no mundo em decorrência da doença, e são previstas pela Organização Mundial da Saúde (OMS) 394.699 mortes e mais de 700 mil novos casos por ano até $2025^{3}$. Para 2014, o Instituto Nacional de Câncer (INCA) estima 15.590 novos casos de câncer cervical no Brasil ${ }^{4}$.

Vários trabalhos demonstram que a distribuição dos tipos de HPV varia de acordo com o grau da lesão, assim como a localização geográfica ${ }^{5-8}$. É consenso que o HPV 16 é o tipo mais frequente globalmente, contudo a contribuição relativa aumenta à medida que o grau da lesão associada é mais alto ${ }^{6}$. Os genótipos HPV 16/18 são responsáveis por $70 \%$ dos casos de câncer cervical invasor (CCI) no mundo. Entretanto, essa fração é maior em regiões mais desenvolvidas, variando de $76 \%$ na América do Norte a $65 \%$ na América do Sul ${ }^{7}$. Essa mesma tendência é observada nas análises que avaliam a distribuição dos genótipos de HPV entre lesão intraepitelial de baixo $(\text { LIE-BG })^{8}$ e alto grau (LIE-AG) ${ }^{7}$.

Os resultados sobre a distribuição dos genótipos de HPV no Brasil são mais modestos, e os estudos são concentrados nos estados da região Sudeste 9 . Em concordância com as observações mundiais, o HPV 16 foi o tipo mais frequente em pesquisas realizadas em diferentes regiões do país ${ }^{10-18}$. Entretanto, a heterogeneidade na distribuição é encontrada principalmente em relação aos demais tipos ${ }^{5-8,19,20}$.

Em estudo piloto na Bahia com mulheres portadoras do vírus da imunodeficiência humana (HIV) e grupo controle, o HPV 16 também foi o mais prevalente em toda a amostra, seguido dos tipos 52, 58, 53, 54, 33 e 51 , apontando para uma possível diferença geográfica da frequência do segundo tipo mais comum ${ }^{18}$.

Diante da escassez de estudos nessa região, o presente trabalho teve como objetivo primário descrever a distribuição dos genótipos de papilomavírus humano em uma amostra de mulheres procedentes do estado da Bahia e secundariamente descrever a frequência de infecções por múltiplos genótipos e avaliar a associação entre genótipos de HPV, faixa etária e resultados cito-histopatológicos.

\section{Métodos}

Foram avaliados retrospectivamente os prontuários das pacientes com genotipagem para HPV que haviam sido submetidas ao exame por apresentar suspeita clínica e/ou cito-histológica de infecção pelo vírus. As mulheres foram atendidas em uma clínica privada, referência para patologia do trato genital inferior na cidade de Salvador, Bahia, entre o período de junho de 2010 e outubro de 2013. O teste de biologia molecular PapilloCheck ${ }^{\circledR}$ (Greiner Bio-One GmbH, Frickenhausen, Alemanha) foi utilizado para identificação simultânea de 24 genótipos (HPV 6, $11,16,18,31,33,35,39,40,42,43,44 / 55,51,52,53$, $56,58,59,66,68,70,73$ e 82). Este ensaio é baseado na detecção de um fragmento do gene E1 do HPV utilizando a tecnologia de DNA-Arrays ${ }^{21}$. As amostras foram provenientes da região cervicovaginal por raspagem, com auxílio de escova do tipo cytobrush. Exames de mulheres procedentes de outros estados negativos ou inconclusivos quanto à infecção por HPV foram excluídos.

Considerando o HPV 16, o mais prevalente $(21,4 \%)^{19}$ entre as mulheres com citologia normal na América do Sul, aceitando-se variação de até cinco pontos percentuais para mais ou para menos e nível de significância de 5\%, foi 
calculado tamanho amostral de 259 casos por intermédio do software WinPEPI. Entretanto, a população a ser estudada também compreenderia, possivelmente, mulheres com diversos achados citológicos. Tendo em vista a proporção de $16,7 \%$ de HPV 16 entre mulheres com LIE-BG ${ }^{8}$ e de $36,9 \%$ para $\mathrm{LIE}-\mathrm{AG}^{20}$, o n seria, respectivamente, 214 ou 358. Optou-se por estudar 350 casos, prevendo maior número de casos de citologia normal e de LIE-BG.

Os resultados do exame histopatológico pela classificação da $\mathrm{OMS}^{22}$ e do exame citopatológico convencional pela terminologia de Bethesda ${ }^{23}$ são adotados pelo serviço onde a pesquisa foi realizada, o que facilita a comparação dos resultados encontrados com outras publicações. Os achados cito-histopatológicos foram classificados em grupos de: achados negativos para neoplasia (exames citopatológico e histopatológico negativos), lesão de baixo grau (achado citopatológico de LIE-BG ou achados histopatológicos de neoplasia intraepitelial cervical grau 1 (NIC1), neoplasia intraepitelial vaginal grau 1 (NIVA1) ou condiloma e lesão de alto grau (englobando achados citopatológicos LIE-AG ou histopatologia com laudo maior igual a NIC2 ou NIVA2). Considerou-se ter lesão o encontro de alteração no exame citopatológico e/ou histopatológico. Foi calculada a razão de prevalência entre ter lesão e o genótipo de HPV. A faixa etária foi categorizada em menor ou maior/igual a 30 anos. Foram calculadas média de idade, proporções segundo estado vacinal para HPV e presença de infecção por HIV.

Os dados foram inseridos no Excel e posteriormente transferidos para o pacote estatístico SPSS (Statistical Package for the Social Sciences), versão 17.0, mediante o qual foram feitas as análises. Foram calculadas a frequência individual dos tipos de HPV e a frequência da infecção por múltiplos genótipos, definida como a presença de dois ou mais tipos virais. Os intervalos de confiança de 95\% (IC95\%) foram calculados para as proporções de infecção por tipo viral e conforme achados cito-histopatológicos. A frequência foi expressa como a proporção do genótipo HPV apenas entre os testes HPV positivos. Foram utilizados os testes do $\chi^{2}$ e exato de Fisher.

Pelo seu caráter exploratório, não era objetivo testar associações, o que foi feito para variáveis específicas unicamente como sondagem para relações promissoras a serem abordadas com desenho confirmatório em iniciativas futuras. O protocolo de estudo foi aprovado pelo Comitê de Ética em Pesquisa do Instituto Médico-Legal Nina Rodrigues (CAAE 19819913.4.0000.5557).

\section{Resultados}

A população inicial de estudo foi constituída por 483 mulheres, das quais 351 tiveram genotipagem positiva para um ou mais tipos de HPV e eram provenientes do estado da Bahia. A amostra foi composta quase que exclusivamente por mulheres com sorologia negativa para HIV (99,4\%, 318/320) e não vacinadas (93,2\%, 275/296). A média etária das pacientes foi de 30 anos com desvio padrão (DP) de 8 , sendo a maioria $(57,4 \%, 198 / 345)$ com idade menor que 30 anos (Tabela 1). Observou-se que $166(58,1 \%)$ mulheres apresentavam achados cito-histopatológicos negativos, 87 (30,4\%), LIE-BG e 33 (11,5\%), LIE-AG.

Dos 351 testes positivos, o tipo oncogênico mais frequente foi o HPV 16, presente em 18,5\% (IC95\% 14,623,0), seguido pelo HPV 56, em 14\% (IC95\% 10,5-18,0) e o HPV 39, em 13,4\% (IC95\% 9,5-16,8). O HPV 18 esteve entre os menos comuns, 5,4\% (IC95\% 3,3-8,3) das mulheres. Entre os tipos não oncogênicos, o HPV 42 $(15,7 \%$; IC95\% 12,0-20,0), HPV 6 (11,4\%; IC 95\% 8,3-15,2) e HPV 44/55 (11,1\%; IC95\% 8-14,9) foram os mais encontrados, enquanto o HPV 11 (2,8\%; IC95\% 1,4-5,2) foi o menos frequente.

A proporção do HPV 16 aumentou com a severidade das anormalidades cito-histopatológicas associadas: de $13,8 \%(12 / 87)$ nas lesões de baixo grau para 42,4\% (14/33) nas lesões de alto grau. Entre as mulheres com LIE-AG cito-histopatológica, o HPV 16 foi o mais frequente, 42,4\% (IC95\% 25,5-60,8), seguido do HPV 56 em 18,2\% (IC 95\% 6,9-35,5). Em mulheres com LIE-BG, o HPV 56 estava presente em 18,4\% (IC95\% 10,9-28,1), seguido pelo HPV 16 em 13,8\% (IC 95\% 7,3-22,8) (Figura 1). Mulheres com menos de 30 anos apresentaram frequência significativamente mais elevada do HPV 16 quando comparadas com aquelas com idade maior/igual a 30 anos (22,2 versus $12,9 \% ; \mathrm{p}=0,01), \mathrm{HPV} 42(19,7$ versus $10,9 \%$; $\mathrm{p}=0,01)$ e HPV $45(6,6$ versus $1,4 \% ; \mathrm{p}=0,01)$ (Figura 2$)$.

Os genótipos de alto risco, HPV 16, 52, 73 e 82, apresentaram associação mais frequente com lesões de baixo ou alto grau, assim como o genótipo de baixo risco, HPV 43 (Tabela 2).

A infecção por múltiplos genótipos de HPV de baixo e/ou alto risco foi detectada em 189 exames $(53,8 \%)$, variando de $2(\mathrm{n}=82,23,4 \%)$ a 10 tipos virais $(\mathrm{n}=1,0,3 \%)$. A média de idade desse grupo foi de 29 anos $(\mathrm{DP}=8)$.

Tabela 1. Caracterização da amostra quanto à vacinação, sorologia por vírus da imunodeficiência humana e aos parâmetros epidemiológicos

\begin{tabular}{lcc}
\hline Característica & $\mathrm{n}$ & $\%$ \\
\hline Não vacinadas $(\mathrm{n}=296)$ & 275 & 93 \\
HIV negativas $(\mathrm{n}=320)$ & 318 & 99,4 \\
Média de idade $(\mathrm{n}=345)$ & 30 anos & 8 (DP) \\
Faixa etária & & \\
$<30$ anos & 190 & 57,4 \\
\hline
\end{tabular}

DP: desvio padrão. HIV: vírus da imunodeficiência humana. As diferenças de $\mathrm{n}$ referem-se às proporções desiguais de participantes com informação disponível. Os dados disponíveis sobre vacina contra HPV, sorologia para HIV e idade foram respectivamente 296,320 e 345 . 


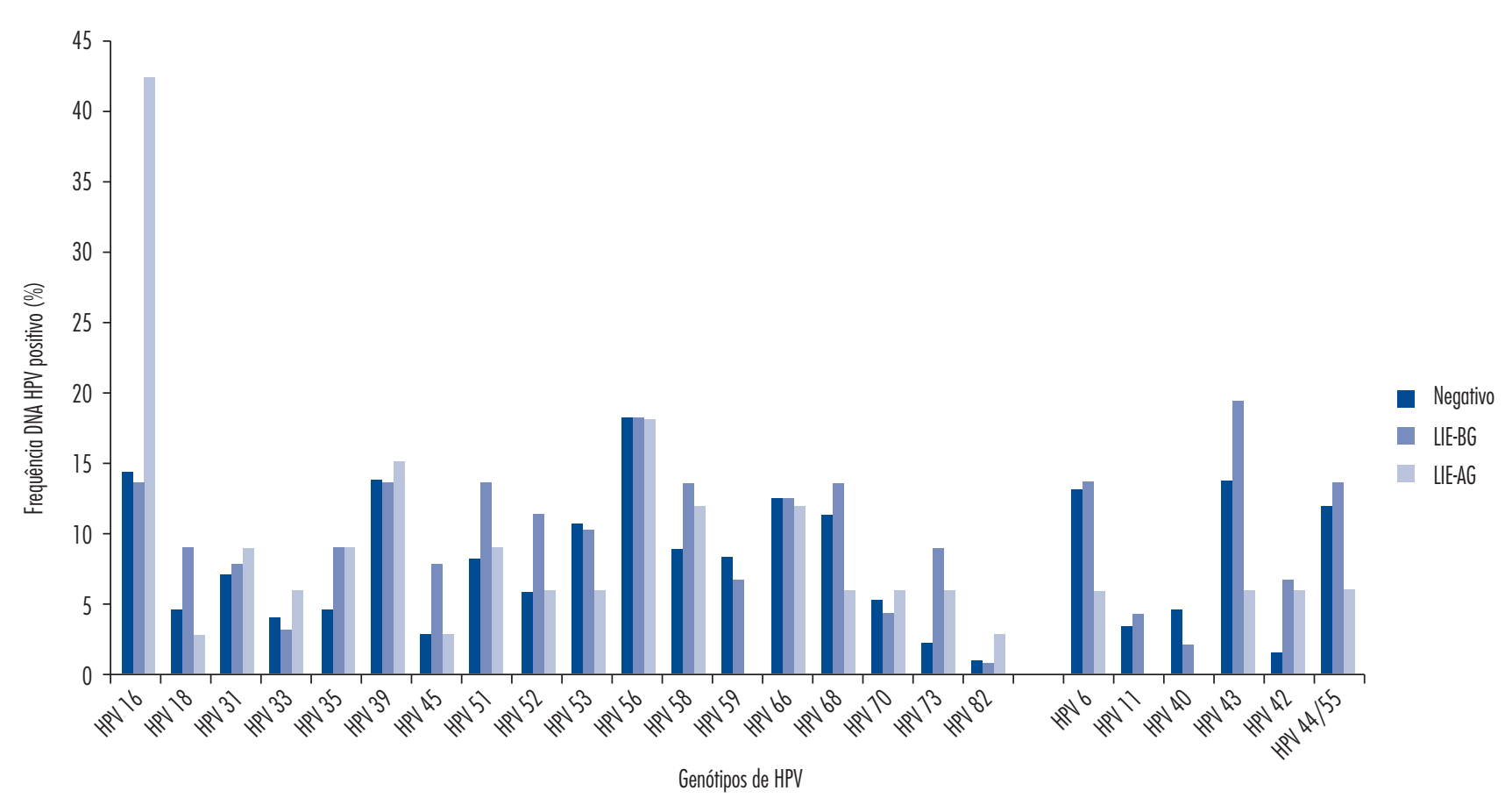

HPV: papilomavírus humano; LIE-BG: lesão intraepitelial de baixo grau; LIE-AG: lesão intraepitelial de alto grau.

Figura 1. Distribuição dos genótipos de papilomavírus humano dentre os achados cito-histológicos negativos, lesão intraepitelial de baixo grau e de alto grau, em amostra de mulheres do estado da Bahia, 2010-2013

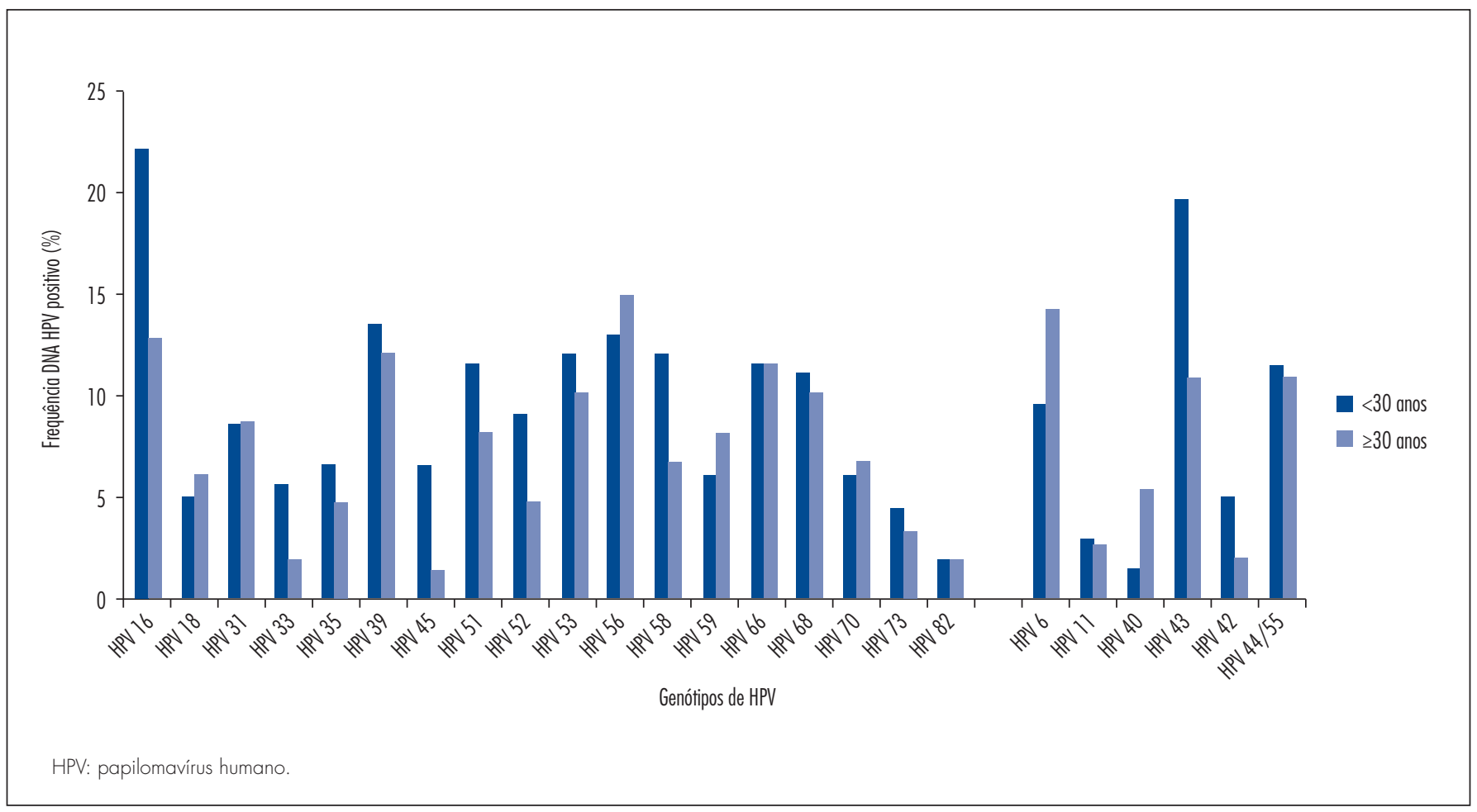

Figura 2. Distribuição dos genótipos de papilomavírus humano conforme faixa etária em amostra de mulheres do estado da Bahia, 2010-2013 
Tabela 2. Razão de prevalência entre ter ou não lesão cito-histológica e genótipo de papilomavírus humano na amostra

\begin{tabular}{|c|c|c|}
\hline Genótipo & $\mathrm{RP}$ & IC95\% \\
\hline HPV 16 & 1,26 & $1,01-157$ \\
\hline HPV 18 & 1,09 & $0,75-1,60$ \\
\hline HPV 31 & 1,1 & $0,80-1,52$ \\
\hline HPV 33 & 0,88 & $0,50-1,57$ \\
\hline HPV 35 & 1,07 & $0,74-1,55$ \\
\hline HPV 39 & 1 & $0,75-1,35$ \\
\hline HPV 45 & 1,2 & $0,82-1,74$ \\
\hline HPV 51 & 1,21 & $0,92-1,59$ \\
\hline HPV 52 & 1,36 & $1,04-1,8$ \\
\hline HPV 53 & 1,04 & $0,77-1,40$ \\
\hline HPV 56 & 1,14 & $0,90-1,47$ \\
\hline HPV 58 & 1,14 & $0,82-1,53$ \\
\hline HPV 59 & 0,84 & $0,53-1,33$ \\
\hline HPV 66 & 0,85 & $0,61-1,19$ \\
\hline HPV 68 & 0,94 & $0,67-1,32$ \\
\hline HPV 70 & 1,17 & $0,83-1,64$ \\
\hline HPV 73 & 1,39 & $1,01-1,90$ \\
\hline HPV 82 & 1,5 & $1,03-2,17$ \\
\hline HPV 6 & 0,91 & $0,64-1,28$ \\
\hline HPV 11 & 0,93 & $0,67-1,29$ \\
\hline HPV 40 & 0,44 & $0,13-1,46$ \\
\hline HPV 42 & 1,12 & $0,87-1,43$ \\
\hline HPV 43 & 1,51 & $1,15-1,99$ \\
\hline HPV 44 & 0,93 & $0,67-1,29$ \\
\hline
\end{tabular}

RP: razão de prevalência; IC95\%: intervalo de confiança de 95\%

A infecção múltipla apenas por tipos de alto risco foi encontrada em 83 mulheres $(41,9 \%)$ e apenas por HPV de baixo risco, em 12 mulheres $(6,1 \%)$.

\section{Discussão}

Nesta amostra, analisamos a frequência dos genótipos de HPV nas infecções únicas e múltiplas em mulheres do estado da Bahia. Nenhum outro estudo semelhante na região foi encontrado, sendo este o primeiro a descrever essa distribuição em mulheres baianas, predominantemente HIV negativas e não vacinadas.

A frequência do HPV 16 teve frequência semelhante às taxas encontradas no Rio Grande do Sul $(18,6 \%)^{11} \mathrm{e}$ em Santa Catarina $(17,2 \%)^{12}$. Essa predominância foi observada entre os diferentes resultados da citologia, dados plenamente concordantes com a literatura mundial e nacional ${ }^{5-8,14-21}$. O HPV 16 é o mais comum nas pesquisas realizadas no Rio Grande do Sul ${ }^{13} \mathrm{Natal}^{10}$, Rio de Janeiro ${ }^{14}$, Caxias do Sul ${ }^{15}$, Distrito Federal ${ }^{16}$ e Ouro Preto $^{17}$. No entanto, apesar de unânimes em relação à maior prevalência do HPV 16, as pesquisas demonstram heterogeneidade entre os demais tipos estudados nas distintas regiões do globo $^{5-8,19,20}$.
Em metanálise com amostras de mulheres apresentando citologias normais, provenientes de cinco continentes, o HPV 16 foi detectado em 22,5\% das mulheres HPV positivas. O segundo genótipo mais comum na prevalência mundial foi o HPV 18, porém com algumas variações geográficas. O HPV 31 foi mais comum na Europa, enquanto o HPV 52 foi especialmente mais frequente na América do Norte, África e Ásia, e o HPV 58 figurou entre os mais encontrados na América Latina 5 . Em âmbito nacional, estudos descrevem o HPV 58 como o segundo mais frequente em $\mathrm{Natal}^{10}$ e em Caxias do Sul ${ }^{15}$, enquanto no Rio de Janeiro, o segundo mais observado foi o HPV $31^{14}$ e em Ouro Preto, o HPV 61 ${ }^{17}$.

Neste estudo, o HPV 18 aparece entre os menos frequentes, discordando da literatura, que o descreve como o segundo genótipo mais encontrado ${ }^{5,7}$. Por outro lado, respaldado pela metanálise da IARC, que o descreve como o quinto mais frequente ${ }^{19}$, esse resultado pode estar relacionado à amostra predominantemente composta por citologias negativas. Ademais, em comparação com carcinoma escamocelular, o HPV 18 é significativamente mais representado nas lesões glandulares e adenocarcinoma cervical ${ }^{24}$. Contudo, nos quatro únicos casos de atipias de células glandulares (AGC) diagnosticados entre nossas pacientes, esse genótipo não foi detectado, o que pode ser devido ao pequeno número de casos de AGC e à baixa reprodutibilidade do diagnóstico citopatológico das alterações glandulares. É importante observar também que, mesmo nos exames citopatológicos de LIE-AG e histopatologia NIC2+, o HPV 18 não esteve entre os dez mais detectados, conforme é descrito na literatura.

O HPV 56 foi o segundo mais detectado em toda a amostra. Os estudos no Brasil $1^{15,16}$ apontam para a maior prevalência do HPV 58, principalmente na região Nordeste do país ${ }^{10}$. Porém, no presente estudo, o HPV 58 foi o oitavo genótipo mais comum.

Nesta amostra, foi observado que a frequência do HPV 16 mostrou-se mais alta à medida que acompanhava o grau de severidade da lesão, resultados estes concordantes com o seu relatado maior potencial oncogênico ${ }^{6}$. O número de casos de CCI atribuíveis a um determinado HPV depende não só da sua prevalência específica naquela população como também do seu potencial carcinogênico, que ocorre em razão da capacidade inerente de cada vírus em desencadear a transformação neoplásica. Foi avaliada a associação entre genótipo específico e ter ou não lesão cito-histopatológica. O HPV 16 apresentou maior associação com a presença de lesão intraepitelial de baixo ou alto grau, assim como os genótipos 52, 73 e 82 .

O HPV 52 encontra-se entre os mais frequentes relacionados ao câncer cervical ${ }^{7,25}$. Os tipos 73 e 82 são menos frequentes e não foram sistematicamente estudados nas metanálises disponíveis. Os tipos mais raros 
de HPV podem estar associados ao câncer cervical por apresentar variabilidade genética, principalmente alterações na sequência na região dos epítopos dos linfócitos T e B da proteína L1, que permitem ao vírus escapar de ser reconhecido pelo sistema imune. Gurgel el al. ${ }^{26}$ investigaram a prevalência de tipos raros encontrados em lesões cervicais de mulheres provenientes do Nordeste do Brasil e observaram que, a despeito da baixa frequência, eles foram o segundo grupo mais encontrado ${ }^{26}$. Novos estudos poderão esclarecer a influência da infecção única por tipos raros de HPV e câncer cervical.

Assim como observado em outro estudo ${ }^{19}$, entre os genótipos de baixo risco, o HPV 42 foi o mais frequente em toda a amostra. É interessante notar que, em comparação ao HPV 42, o HPV 6 apresentou frequência bem menor entre as diversas lesões. Porém, nas coletas cervicovaginais de mulheres portadoras de condilomas vulvar à histopatologia, o HPV 6 foi observado em taxas superiores ao HPV 42. Esse dado está em concordância com a observação da importante contribuição do HPV 6, que, associado ao HPV 11, é responsável por $90 \%$ das verrugas genitais. Apesar disso, o HPV 11 foi um dos menos frequentes em toda amostra.

Diante de todos os dados apresentados, é preciso ter cautela quando se interpretar os resultados obtidos por diferentes testes moleculares. Eles apresentam sensibilidades analíticas diferentes, particularmente em amostras com baixa carga viral, comuns em achados citopatológicos negativos ${ }^{21}$. Isso ocorre principalmente com genótipos menos comuns. Apesar de as metanálises calcularem a prevalência específica somente dos tipos detectados em cada estudo, alguns genótipos podem ter sua estimativa comprometida a depender do teste de genotipagem utilizado em determinada região ${ }^{5}$.

A amostra incluída no estudo apresentou média etária de 30 anos, sendo a maioria com menos de 30 anos. Essa observação é respalda pelos estudos da história natural da infecção pelo HPV. A aquisição do vírus ocorre após o início da vida sexual e decai com a idade, principalmente após 30-35 anos, seja pelo clearance do vírus, mudança nos hábitos sexuais ou imunidade adquirida naturalmente pelas infecções prévias ${ }^{27}$. Foi também observado que as mulheres mais jovens apresentavam significativamente maior frequência da infecção por múltiplos genótipos do que as na faixa etária acima dos 30 anos, similar a outros estudos ${ }^{17}$.

A infecção por múltiplos genótipos ganhou importância com o advento da vacina contra infecção pelo HPV. É aventado que a introdução dessa imunização talvez possa modificar o equilíbrio já estabelecido da distribuição dos genótipos ${ }^{28}$. Nesta amostra, a taxa de infecção por múltiplos genótipos foi semelhante à relatada por Adler et al. ${ }^{29}$ (68\%), porém muito superior ao encontrado em Ouro Preto $(27 \%)^{17}$ e Rio Grande do Sul $(16,7 \%)^{13}$. A prevalência da infecção pelo HPV e das infecções múltiplas descritas nos estudos entre 1990 e 2010 aumentou significativamente ${ }^{25}$. O aumento da sensibilidade dos métodos moleculares permite a amplificação de um maior número de genótipos e, portanto, interfere na frequência encontrada da infecção pelo HPV, seja única ou por múltiplos genótipos.

Entre as limitações deste estudo, o caráter da coleta retrospectiva de dados em prontuários não permitiu que os exames cito-histopatológicos e o teste DNA/HPV fossem realizados em um mesmo momento. Além disso, alguns dados não puderam ser coletados por não estarem disponíveis. É interessante também observar que a população do estudo representa as mulheres de serviços de referência para patologia cervical, em que é esperada maior prevalência da infecção pelo HPV. Entretanto, na prática médica atual, são essas mulheres que apresentam indicação para submeter-se aos exames de biologia molecular diante da suspeita de lesão HPV induzida ou no controle pós-tratamento de lesões intraepiteliais. Portanto, apesar de a presente amostra não poder ser diretamente comparada aos estudos que avaliam a prevalência dos genótipos em mulheres sem histórico de infecção, tem validade externa para mulheres com esse perfil. A proposta do estudo de avaliar a distribuição dos genótipos de HPV com o uso do teste PapilloCheck ${ }^{\circledR}$, disponível comercialmente na prática clínica, foi alcançada. Entretanto, devemos ponderar as comparações com os estudos descritos na literatura utilizando testes moleculares de detecção de HPV com sensibilidade e especificidade diferentes. Por sua vez, esses resultados corroboram outros estudos no Brasil que observam uma variabilidade da distribuição dos diversos genótipos de HPV em mulheres com lesões pré-neoplásicas ou câncer e preenchem uma lacuna do conhecimento no estado da Bahia.

Em conclusão, assim como em outras regiões do Brasil e do mundo, o HPV 16 foi o tipo mais frequente, corroborando a indicação da vacinação contra esse genótipo. Entre os genótipos oncogênicos, os HPV 56 e HPV 39 foram o segundo e o terceiro mais frequentes, entretanto o HPV 18 esteve entre os menos comuns. Entre os HPV não oncogênicos, os HPV 42, 6 e 44/55 foram os mais frequentes, enquanto o HPV 11 foi o menos frequente. Esses dados estão em concordância com a literatura em relação à existência de uma heterogeneidade na distribuição dos diversos genótipos. Novos trabalhos com o uso de testes moleculares padronizados e abrangendo diferentes regiões do estado permitirão definir com maior precisão a frequência dos genótipos de HPV na Bahia, o que será de relevância para o planejamento de políticas públicas relacionadas ao diagnóstico e à prevenção das infecções e lesões induzidas por esse vírus. 
1. Steben $M$, Duarte-Franco E. Human papillomavirus infection: epidemiology and pathophysiology. Gynecol Oncol. 2007;107(2 Suppl 1):S2-5.

2. Walboomers JM, Jacobs MV, Manos MM, Bosch FX, Kummer $J A$, Shah KV, et al. Human papillomavirus is a necessary cause of invasive cervical cancer worldwide. J Pathol. 1999;189(1):12-9.

3. WHO/ICO Information Centre on HPV and Cervical Cancer (HPV Information Centre). Human papillomavirus and related cancers. Summary Report Update. Barcelona; 2010.

4. Brasil. Ministério da Saúde. Instituto Nacional de Câncer José Alencar Gomes da Silva [Internet]. Câncer do colo do útero. Rio de Janeiro: INCA; 2013 [citado 2014 Jan 5]. Disponível em: http://www2.inca. gov.br/wps/wcm/connect/tiposdecancer/site/home/colo_utero

5. Bruni L, Diaz M, Castellsagué X, Ferrer E, Bosch FX, de Sanjosé S. Cervical human papillomavirus prevalence in 5 continents: meta-analysis of 1 million women with normal cytological findings. J Infect Dis. 2010;202(12):1789-99.

6. Guan P, Howell-Jones R, Li N, Bruni L, de Sanjosé S, Franceschi $S$, et al. Human papillomavirus types in 115,789 HPV-positive women: a meta-analysis from cervical infection to cancer. Int J Cancer. 2012;131(10):2349-59.

7. Smith JS, Lindsay L, Hoots B, Keys J, Franceschi S, Winer R, et al. Human papillomavirus type distribution in invasive cervical cancer and high-grade cervical lesions: a meta-analysis update. Int J Cancer. 2007;121(3):621-32.

8. Clifford GM, Rana RK, Franceschi S, Smith JS, Gough G, Pimenta $J M$. Human papillomavirus genotype distribution in low-grade cervical lesions: comparison by geographic region and with cervical cancer. Cancer Epidemiol Biomarkers Prev. 2005; 14(5): 1 157-64.

9. Ayres ARG, Silva GA. Cervical HPV infection in Brazil: systematic review. Rev Saúde Pública. 2010;44(5):963-74.

10. Medeiros Fernandes TA, de Vasconcellos Meissner R, Bezerra LF, Azevedo PR, Fernandes JV. Human papillomavirus infection in women attended at a cervical cancer screening service in Natal, Brazil. Braz J Microbiol. 2008;39(3):573-8.

11. Rosa MI, Fachel JMG, Rosa DD, Medeiros LR, Igansi CN, Bozzetti MC. Persistence and clearance of human papillomavirus infection: a prospective cohort study. Am J Obstet Gynecol. 2008;199(6):617.e1-7.

12. Krambeck WM, Cadidé RM, Dalmarco EM, De Cordova CMM. HPV detection and genotyping as an earlier approach in cervical cancer screening of the female genital tract. Clin Exp Obstet Gynecol [Internet]. 2008 [cited 2013 Oct 30];35(3): 175-8. Available from: http://cat.inist.fr/?aModele=afficheN\&cpsidt=20600223

13. Coser J, da Rocha Boeira TR, Simon D, Kazantzi Fonseca AS, Ikuta $\mathrm{N}$, Lunge VR. Prevalence and genotypic diversity of cervical human papillomavirus infection among women from an urban center in Brazil. Genet Mol Res. 2013;12(4):4276-85.

14. Oliveira LH, Ferreira MD, Augusto EF, Melgaço FG, Santos LS, Cavalcanti SM, et al. Human papillomavirus genotypes in asymptomatic young women from public schools in Rio de Janeiro, Brazil. Rev Soc Bras Med Trop. 2010;43(1):4-8.

15. Paesi S, Serafini EP, Barea F, Madi SR, Echeverrigaray S. High prevalence of human papillomavirus type 58 in patients with cervical pre-malignant lesions in southern Brazil. J Med Virol. 2009;81(7):1270-5.
16. Camara GN, Cerqueira DM, Oliveira AP, Silva EO, Carvalho LG Martins CR. Prevalence of human papillomavirus types in women with pre-neoplastic and neoplastic cervical lesions in the Federal District of Brazil. Mem Inst Oswaldo Cruz. 2003;98(7):879-83.

17. Miranda PM, Pitol BC, Moran MS, Silva NN, Felix PM, Lima-Filho $J L$, et al. Human papillomavirus infection in Brazilian women with normal cervical cytology. Genet Mol Res. 2012;1 1 (2):1752-61.

18. Queiroz C, Travassos AG, Studart E, Araújo Filho JB, Sarno CK, Pinheiro CC. Prevalence of human Papilloma Virus in HIV-positive and HIV-negative patients in the State of Bahia: a pilot study. Brazilian J Infect Dis. 2004;8(5):356-62.

19. Clifford GM, Gallus S, Herrero R, Muñoz N, Snijders PJ, Vaccarella $S$, et al. Worldwide distribution of human papillomavirus types in cytologically normal women in the International Agency for Research on Cancer HPV prevalence surveys: a pooled analysis. Lancet. 2005;366(9490):991-8.

20. Clifford GM, Smith JS, Aguado T, Franceschi S. Comparison of HPV type distribution in high-grade cervical lesions and cervical cancer: a meta-analysis. Br J Cancer. 2003;89(1):101-5.

21. Dalstein V, Merlin S, Bali C, Saunier M, Dachez R, Ronsin C. Analytical evaluation of the PapilloCheck test, a new commercial DNA chip for detection and genotyping of human papillomavirus. J Virol Methods. 2009; 156(1-2):77-83.

22. Tavassoli FA, Devilee P. Pathology and genetics of tumours of the breast and female genital organs [Internet]. Lyon: IARC Press; 2003 [cited 2014 May 28]. (World Health Organization Classification Tumours, 5). Available from: http://www.iarc.fr/en/publications/ pdfs-online/pat-gen/bb4/BB4.pdf

23. Solomon D, Davey D, Kurman R, Moriarty A, O'Connor D, Prey $M$, et al. The 2001 Bethesda System: terminology for reporting results of cervical cytology. JAMA. 2002;287(16):21 14-9.

24. Clifford G, Franceschi S. Members of the human papillomavirus type 18 family (alpha-7 species) share a common association with adenocarcinoma of the cervix. Int J Cancer. 2008;122(7): 1684-5.

25. Li N, Franceschi S, Howell-Jones R, Snijders PJ, Clifford GM. Human papillomavirus type distribution in 30,848 invasive cervical cancers worldwide: variation by geographical region, histological type and year of publication. Int J Cancer. 2011 ; 128(4):927-35.

26. Gurgel AP, Chagas BS, do Amaral CM, Albuquerque EM, Serra IG, Silva Neto JC, et al. Prevalence and genetic variability in capsid L1 gene of rare human papillomaviruses (HPV) found in cervical lesions of women from North-East Brazil. Biomed Res Int. 2013;2013:546354.

27. Moscicki AB, Schiffman M, Kjaer S, Villa LL. Chapter 5: Updating the natural history of HPV and anogenital cancer. Vaccine. 2006;24(Suppl 3):S3/42-51.

28. Pons-Salort M, Letort V, Favre M, Heard I, Dervaux B, Opatowski $L$, et al. Exploring individual HPV coinfections is essential to predict HPV-vaccination impact on genotype distribution: a model-based approach. Vaccine. 2013;31(8):1238-45.

29. Adler D, Laher F, Wallace M, Grzesik K, Jaspan H, Bekker LG, et al. High rate of multiple concurrent Human Papillomavirus infections among HIV-uninfected South African Adolescents. J Immunol Tech Infect Dis. 2013;2:1. 